University of Texas at El Paso

ScholarWorks@UTEP

6-2016

\title{
How Resilient Modulus of a Pavement Depends on Moisture Level: Towards a Theoretical Justification of a Practically Important Empirical Formula
}

\author{
Pedro Barragan Olague \\ The University of Texas at El Paso, pabarraganolague@miners.utep.edu \\ Olga Kosheleva \\ The University of Texas at El Paso, olgak@utep.edu \\ Vladik Kreinovich \\ The University of Texas at El Paso, vladik@utep.edu
}

Follow this and additional works at: https://scholarworks.utep.edu/cs_techrep

Part of the Computer Sciences Commons

Comments:

Technical Report: UTEP-CS-16-36

\section{Recommended Citation}

Olague, Pedro Barragan; Kosheleva, Olga; and Kreinovich, Vladik, "How Resilient Modulus of a Pavement Depends on Moisture Level: Towards a Theoretical Justification of a Practically Important Empirical Formula" (2016). Departmental Technical Reports (CS). 1025.

https://scholarworks.utep.edu/cs_techrep/1025

This Article is brought to you for free and open access by the Computer Science at ScholarWorks@UTEP. It has been accepted for inclusion in Departmental Technical Reports (CS) by an authorized administrator of ScholarWorks@UTEP. For more information, please contact Iweber@utep.edu. 


\title{
How Resilient Modulus of a Pavement Depends on Moisture Level: Towards a Theoretical Justification of a Practically Important Empirical Formula
}

\author{
Pedro Barragan Olague, Olga Kosheleva, \\ and Vladik Kreinovich \\ University of Texas at El Paso \\ $500 \mathrm{~W}$. University \\ El Paso, TX 79968, USA \\ pabarraganolague@miners.utep.edu \\ olgak@utep.edu,vladik@utep.edu
}

\begin{abstract}
Resilient modulus is a mechanical characteristic describing the stiffness of a pavement. Its value depends on the moisture level. In pavement construction, it is important to be able, knowing the resilient modulus corresponding to one moisture level, to predict resilient modulus corresponding to other moisture levels. There exists an empirical formula for this prediction. In this paper, we provide a possible theoretical explanation for this empirical formula.
\end{abstract}

\section{Formulation of THE PRoblem}

What is a resilient modulus: a brief reminder. A road pavement must be sufficiently stiff: when heavy trucks pass over the pavement at a high speed, the pavement's deformation must be within certain (small) bounds.

In mechanics in general, a usual characteristic of stiffness is modulus, the ratio of stress (force per area) to deformation. For a fixed force, the larger the modulus, the smaller the deformation.

Force applied to the pavement mostly comes from the fast moving vehicles. The effect of such a force can be characterized by a special type of modulus called resilient modulus.

Dependence on moisture is important. To increase stiffness, the pavement is compacted. After the compaction, it is necessary to check whether the resilient modulus has reached the desired value, to see whether we need to perform additional compaction (or add an additional pavement layer) to increase stiffness to the desired level.

Ideally, we should make this decision right away, when the equipment is still in the road segment. The problem is that after the compaction, it often takes several days for the moisture content to reach the equilibrium. During these days, the equipment has been moved to new road segments. So, if we perform the measurements only after this equilibrium is attained, and it turns out that further work is needed, we will then need to move the equipment back, which requires extra time and extra expenses.

It is therefore desirable, given the resilient modulus under the current moisture level, to predict the future equilibrium value of the resilient modulus corresponding to the future equilibrium level of moisture.

An empirical formula that describes the dependence of resilient modulus on the moisture level. The moisture level is usually characterized by the degree of saturation $S$, which is defined as a ratio of the volume of water to the overall volume of the empty space in the pavement:

- no moisture at all corresponds to $S=0.0$, while

- the situation where all the empty spaces between the pavement' solid pieces are filled with water corresponds to $S=1.0$.

There exists an empirical formula that describes the dependence of the resilient modulus $M(S)$ on the moisture level $S$ :

$$
\ln \left(\frac{M(S)}{M\left(S_{0}\right)}\right)=a+\frac{b-1}{1+\exp \left(\beta-k \cdot\left(S-S_{0}\right)\right)}
$$

for some moisture level $S_{0}$; see, e.g., [4].

Comment. Substituting $S=S_{0}$ into the formula (1), we conclude that

$$
0=a+\frac{b-a}{1+\exp (\beta)}=0
$$

hence

$$
\frac{b-a}{1+\exp (\beta)}=-a
$$

and

$$
1+\exp (\beta)=-\frac{b-a}{a}=1-\frac{b}{a} .
$$

Thus, we have $\exp (\beta)=-b / a$, and $\beta=\ln (-b / a)$.

It is desirable to come up with a theoretical foundation for this empirical formula. The problem of estimating how moisture level affects resilient modulus is practically important. It is therefore desirable to make related conclusions with as much reliability as possible.

In general, purely empirical formulas are less reliable: without a theoretical justification, the fact that the formula was observed to be true in several situations does not necessarily 
mean that it will also be true in other situations. It is therefore desirable to come up with a theoretical justification for the above empirical formula (1).

Such a justification will be provided in this paper. This explanation will be based on the ideas similar to what we used to explain a related empirical formula - a formula that describes the dependence of resilient modulus on the stress values [2].

\section{Main Idea Behind the Proposed Explanation}

Scale invariance. The numerical value of a physical quantity depends on the choice of a measuring unit. If we use a different measuring unit, then all the numerical values change. Specifically, if we replace the original measuring unit with a new unit which is $\lambda$ times smaller, then all numerical values get re-scaled: namely, they are multiplied by $\lambda$ :

$$
x \rightarrow x^{\prime}=\lambda \cdot x .
$$

For example, if we originally measures lengths in meters, and decided to use instead a new unit, centimeter, which is $\lambda=100$ times smaller, then all the length values are multiplied by 100: e.g., $3 \mathrm{~m}$ becomes $3 \cdot 100=300 \mathrm{~cm}$.

In most physical situations, the choice of a measuring unit is rather irrelevant. Thus, the corresponding formulas should not change if we use different units.

Of course, if we describe a dependence $y=f(x)$ and we change a unit for measuring $x$, then we may have to according also change the measuring unit for $y$; then, in the new units for $x$ and $y$, the dependence should look exactly the same as in the old units.

Shift invariance. For some quantities, the starting point can be arbitrarily chosen. For example, we can start measuring time at any moment - we can start at year 0 , or we can take year 2016 as year 0 .

If we replace the original starting point with a new one which is $x_{0}$ unit earlier, then for the same moment of time, the original numerical value $x$ gets replaced by a new shifted value $x^{\prime}=x+x_{0}$.

It is therefore reasonable to require that for such quantities, if we change the starting point, the resulting dependencies should remain the same.

Let us use these invariances. Let us use these two invariances to derive the desired empirical formula (1).

\section{Analysis of the Problem And the Resulting DERIVATION OF THE EMPIRICAL FORMULA}

Simplified problem. Let us start our analysis with the following simplified problem. Our general problem is to find how the resilient modulus $M(S)$ depend on the moisture level $S$. Instead of considering all possible moisture levels $S$, let us fix two values $S_{1}$ and $S_{2}$, and analyze how:

- if we know the resilient modulus $M\left(S_{1}\right)$ corresponding to moisture level $S_{1}$,

- then we can predict the resilient modulus $M\left(S_{2}\right)$ corresponding to the moisture level $S_{2}$.
Since the two moisture levels are fixed, the predicted value $M\left(S_{2}\right)$ depends only on the value $M\left(S_{1}\right)$, i.e.,

$$
M\left(S_{2}\right)=f\left(M\left(S_{1}\right)\right)
$$

for an appropriate function $f(x)$. Which function $f(x)$ should we choose?

Let us use scale-invariance to solve the simplified problem. If we change the unit for measuring the resilient modulus to a new unit which is $\lambda$ times smaller, then both numerical values $M\left(S_{1}\right)$ and $M\left(S_{2}\right)$ get multiplied by $\lambda$, so we get new numerical values

$$
M\left(S_{1}\right) \rightarrow M^{\prime}\left(S_{1}\right)=\lambda \cdot M\left(S_{1}\right)
$$

and

$$
M\left(S_{2}\right) \rightarrow M^{\prime}\left(S_{2}\right)=\lambda \cdot M\left(S_{2}\right) .
$$

The requirement that the dependence

$$
M\left(S_{2}\right)=f\left(M\left(S_{1}\right)\right)
$$

does not change under this re-scaling implies that

$$
M^{\prime}\left(S_{2}\right)=f\left(M^{\prime}\left(S_{1}\right)\right)
$$

i.e.,

$$
\lambda \cdot M\left(S_{2}\right)=f\left(\lambda \cdot M\left(S_{1}\right)\right) .
$$

Substituting

$$
M\left(S_{2}\right)=f\left(M\left(S_{1}\right)\right)
$$

into this formula, we conclude that

$$
f\left(\lambda \cdot M\left(S_{1}\right)\right)=\lambda \cdot f\left(M\left(S_{1}\right)\right)
$$

for all possible values $\lambda>0$ and $M\left(S_{1}\right)>0$. In particular, for any real number $x$, we can take $\lambda=x$ and $M\left(S_{1}\right)=1$, in which case we get

$$
f(x)=x \cdot f(1)
$$

i.e.,

$$
f(x)=c \cdot x
$$

for some constant $c$.

Thus, we conclude that for every two moisture levels $S_{1}$ and $S_{2}$, the corresponding values $M\left(S_{1}\right)$ and $M\left(S_{2}\right)$ of the resilient modulus are connected by the formula

$$
M\left(S_{2}\right)=c\left(S_{1}, S_{2}\right) \cdot M\left(S_{1}\right),
$$

for some coefficient $c\left(S_{1}, S_{2}\right)$ depending on the moisture levels.

Let us find the dependence $c\left(S_{1}, S_{2}\right)$.

We can use different starting points for measuring moisture level. Moisture level is a unit-less quantity, there is no easy way to change a measuring unit. However, it turns out that the starting point for measuring moisture level can be chose differently.

We can start with the absolute zero moisture level. This level is difficult to obtain in practice, but the corresponding 
amount of moisture is what is measured by the current nuclear magnetic resonance-based measuring instruments.

Alternatively, we can try to dry out the piece of pavement and measure the original moisture level by how much water evaporated. In this cases, we measure the moisture level not against the ideal no-moisture situation, but against a dry equilibrium state, in which some water remains (to completely eliminate all water, we need to warm the pavement so much that it may be destroyed).

It is therefore reasonable to require that the formulas should not change if we change the starting point for measuring moisture level, i.e., if we replace the original numerical value $S$ by the new value $S^{\prime}=S+S_{0}$.

How to use shift-invariance: first idea. A seemingly natural idea is to require that the dimensionless coefficient $c\left(S_{1}, S_{2}\right)$ does not change if we shift all the values, i.e., if

$$
c\left(S_{1}+S_{0}, S_{2}+S_{0}\right)=c\left(S_{1}, S_{2}\right)
$$

for all $S_{1}, S_{2}$, and $S_{0}$. In particular, for $S_{0}=-S_{2}$, we conclude that

$$
c\left(S_{1}, S_{2}\right)=c\left(S_{1}-S_{2}, 0\right),
$$

i.e., that the value $c\left(S_{1}, S_{2}\right)$ depends only on the difference between moisture levels:

$$
c\left(S_{1}, C_{2}\right)=C\left(S_{1}-S_{2}\right),
$$

where we denoted

$$
C(S) \stackrel{\text { def }}{=} c(S, 0)
$$

In this case, for every $S_{1}$ and $S_{2}$, we have

$$
M\left(S_{1}\right)=C\left(S_{1}\right) \cdot M(0)
$$

and

$$
M\left(S_{1}+S_{2}\right)=C\left(S_{2}\right) \cdot M\left(S_{1}\right)
$$

and thus,

$$
M\left(S_{1}+S_{2}\right)=C\left(S_{2}\right) \cdot C\left(S_{1}\right) \cdot M(0) .
$$

On other other hand, we can directly apply the shift by $S_{1}+S_{2}$, then we get

$$
M\left(S_{1}+S_{2}\right)=C\left(S_{1}+S_{2}\right) \cdot M(0) .
$$

By comparing the two formulas for $M\left(S_{1}+S_{2}\right)$, we conclude that

$$
C\left(S_{1}+S_{2}\right)=C\left(S_{2}\right) \cdot C\left(S_{1}\right) .
$$

In physics, most dependencies are continuous. It is therefore reasonable to require that the dependence of $M(S)$ on $S$ is continuous. Thus, the dependence of

$$
C(S)=\frac{M(S)}{M(0)}
$$

on $S$ should also be continuous. All continuous functions satisfying the functional equation

$$
C\left(S_{1}+S_{2}\right)=C\left(S_{2}\right) \cdot C\left(S_{1}\right)
$$

are known: they all have the form

$$
C(S)=\exp (k \cdot S)
$$

for some parameter $k$; see, e.g., [1]. Thus, we get a simple formula

$$
M(S)=\exp (k \cdot S) \cdot M(0)
$$

Problem with the first idea. The problem is that the resulting simple formula

$$
M(S)=\exp (k \cdot S) \cdot M(0)
$$

does not fit well with empirical data. Thus, we need to use invariances in a more subtle way.

Second idea. The second idea is that if we know $M(S)$ and some moisture-independent value $M$ (e.g., the largest possible value of resilient modulus corresponding to all possible moisture levels), then we should be able to reconstruct $M\left(S+S_{0}\right)$. In other words, we should have

$$
M\left(S+S_{0}\right)=f(M(S), M)
$$

for some function $f(x, y)$ depending only on $S_{0}$.

The dependence $f(x, y)$ should be scale-invariant. Let us take into account that we may have different procedures for measuring $M$ and $M(S)$, and thus, in principle, we may have two different measuring units used in these measurements. It is therefore reasonable to require that we can change the units of both quantities and still get the same dependence - after, possibly, appropriately re-scaling the resulting value

$$
M\left(S+S_{0}\right) .
$$

In other words, for every two factors $\lambda$ and $\mu$ that describe the re-scaling of $M(S)$ and $M$, we have a factor $\nu(\lambda, \mu)$ for which, for all $M(S)$ and $M$,

$$
M\left(S+S_{0}\right)=f(M(S), M)
$$

implies that

$$
f(\lambda \cdot M(S), \mu \cdot M)=\nu(\lambda, \mu) \cdot f(M(S), M) .
$$

It is known that every continuous function $f(x, y)$ with this property has the form

$$
f(x, y)=A \cdot x^{\alpha} \cdot y^{\beta}
$$

for some real numbers $\alpha$ and $\beta$; see, e.g., [1], [2]. Thus, we conclude that

$$
M\left(S+S_{0}\right)=A \cdot(M(S))^{\alpha} \cdot M^{\beta} .
$$

Substituting $M(S)=C(S) \cdot M$ and

$$
M\left(S+S_{0}\right)=C\left(S+S_{0}\right) \cdot M
$$

into this formula, we conclude that

$$
C\left(S+S_{0}\right) \cdot M=A \cdot(C(S))^{\alpha} \cdot M^{\alpha} \cdot M^{\beta} .
$$


In particular, for $M=1$, we get

$$
C\left(S+S_{0}\right)=A \cdot(C(S))^{\alpha},
$$

where $A$ and $\alpha$ depend on $S_{0}$.

By taking logarithms of both sides, we conclude that for the function

$$
L(S) \stackrel{\text { def }}{=} \ln (C(S))
$$

we get

$$
L\left(S+S_{0}\right)=a\left(S_{0}\right)+\alpha\left(S_{0}\right) \cdot L(S),
$$

where

$$
a\left(S_{0}\right) \stackrel{\text { def }}{=} \ln \left(A\left(S_{0}\right)\right) .
$$

So, the value $L\left(S+S_{0}\right)$ is obtained from $L(S)$ by applying a linear transformation

$$
L(S) \rightarrow a\left(S_{0}\right) \cdot \alpha\left(S_{0}\right) \cdot L(S) .
$$

Problem with the second idea. Functions satisfying the above functional equation are also known known; see, e.g., [5]. Alas, these functions are also not a perfect fit for the empirical data.

Final idea. Since linear transformations are not sufficient, let us consider the possibility that the transformation from $L(S)$ to $L\left(S+S_{0}\right)$ may be non-linear. To be precise, we are looking for a group of transformations - i.e., set of transformations which is closed under composition and taking the inverse that:

- includes all linear transformations and

- that is not too large - e.g., is finite-dimensional (meaning that each transformation can be uniquely determined by specifying values of finitely many parameters).

It turns out that, under certain reasonable continuity requirements, all such transformations are known (see, e.g., [3], [5], [6]): they are fractional-linear transformations

$$
L(S) \rightarrow \frac{a \cdot L(S)+b}{c \cdot L(S)+d} .
$$

The corresponding functions $L(S)$ for which

$$
L\left(S+S_{0}\right)=\frac{a\left(S_{0}\right) \cdot L(S)+b\left(S_{0}\right)}{c\left(S_{0}\right) \cdot L(S)+d\left(S_{0}\right)}
$$

are also known [5]: under reasonable monotonicity conditions, they get exactly the form (1). Thus, we indeed get the desired justification for the above empirical formula.

\section{ACKNOWLEDGMENTS}

This work was supported in part by the National Science Foundation grants HRD-0734825 and HRD-1242122 (CyberShARE Center of Excellence) and DUE-0926721, and by an award "UTEP and Prudential Actuarial Science Academy and Pipeline Initiative" from Prudential Foundation.

\section{REFERENCES}

[1] J. Aczél and J. Dhombres, Functional Equations in Several Variables, Camridge University Press, Cambridge, UK, 2008.

[2] P. Barragan Olague, S. Nazarian, V. Kreinovich, A. Gholamy, and M. Mazari, "How to Estimate Resilient Modulus for Unbound Aggregate Materials: A Theoretical Explanation of an Empirical Formula", Proceedings of the 2016 World Conference on Soft Computing, Berkeley, California, May 22-25, 2016, pp. 203-207.

[3] V. M. Guillemin and S. Sternberg, "An algebraic model of transitive differential geometry", Bulletin of the American Matjematical Society, 1964, Vol. 70, No. 1, pp. 16-47.

[4] N. N. Khoury and M. M. Zaman, "Correlation between resilient modulus, moisture variation, and soil suction for subgrade soils", Transportation Research Record: Journal of Transportation Research Board, 2004, No. 1874, pp. 99-107.

[5] H. T. Nguyen and V. Kreinovich, Applications of continuous mathematics to computer science, Kluwer, Dordrecht, 1997.

[6] I. M. Singer and S. Sternberg, Infinite groupsof Lie and Cartan, Part I, Journal d'Analyse Mathematique, 1965, Vol. 15, pp. 1-113. 